\title{
Revelations of the Blindness Prevention Modes in India and Cuba for China
}

\author{
Qing Xu, Haiyang Wu, Baili Zhong \& Baojie Hou \\ Department of Ophthalmology, China Armed Police General Hospital \\ Beijing 100039, China \\ E-mail: xuqingwjz@vip.sina.com
}

\begin{abstract}
Though the blindness prevention program is highly effective, but comparing with developed countries and part of developing countries, the gap is still obvious, and the blindness prevention mode and the implementation measures are still influencing the blindness prevention process. The blindness prevention performance of India and Cuba is notable, and the advantages of the Indian cross subsidization method and the Cuba government investment mode should be used for references in China.
\end{abstract}

Keywords: Blindness prevention, Cataract, India, Cuba

The blindness is divided into the avoidable blindness and the preventable blindness in the WHO. The avoidable blindness means the diseases which could be cured by medicines or surgeries, such as cataract which is the major cause of vision loss in the world, $39 \%$ of all blinds. The preventable blindness means the blindness which could not be reversed, but can be controlled by the medical interference, such as glaucoma, diabetes retina pathological changes and so on.

For a long time, the blindness prevention is very important in the department of ophthalmology, and though numerous oculists have paid large effort for the blindness prevention in China, but the situation is still far from being satisfactory, and comparing with developed countries, the blindness prevention of China is still lagged, and even comparing with some developing countries such as India and Cuba, the gap is still large. In recent two years, through the practical works in India and Cuba, the author felt much, and the blindness prevention program of China should use the advantages in India and Cuba for references, and be changed as follows.

\section{India blindness prevention mode}

India is one of the countries with the prominent performance of blindness prevention, and its amount of cataract surgery rate (the cataract surgery rate in one million people each year) is 3100, eight times of China's (Xu, 2008, P.1-3). Different blindness prevention effects occurred in similar economic states, and following characteristics exist in Indian blindness prevention mode.

\subsection{Positioning screening}

In this mode, large ophthalmologic hospitals radiate many sub-centers by the network information management, as the center, and many screening positions are established in the schools of some villages, and the doctors will be dispatched periodically to screen and treat eye diseases on the spot, and the patients who could not been treated will be moved to the ophthalmologic hospitals for the surgery treatment. $50 \%$ of cataract patients come from that. The large-scale positioning screening could provide convenient and quick medical approaches to solve the mess that poor and far patients could not be treated. And all screenings are free.

\subsection{Free operation}

Because of the faith, many private hospitals in India could provide free surgeries for cataract patients. The Aravind Ophthalmologic Hospital is a large private ophthalmologic hospital in the South of India, with 3000 beds, and in 2006, there were 0.19 million cataract patients to be treated, and $2 / 3$ of them were free. The hospital adopted the method of cross subsidization, i.e. 1/3 patients' charge with payable ability would be enhanced for the other $2 / 3$ patients. Like China, many people in India are too poor to pay expensive medical charge, and by this method of cross subsidization, many patients could obtain the free surgeries to cure the cataract. Those patients paying for the surgeries will feel calm because they could help others. 


\subsection{Manual small incision cataract surgery}

In the cataract blindness prevent surgery, the hospitals need saving the costs for free surgeries, so they may not invest high-cost equipments and technologies, and 3manual small incision cataract surgery with cheap cost and equipments just could possess this advantage, and for a good doctor, the after-operation effect of the small incision operation is almost same with the phacoemulsification (Ruit S, Tabin G, 2007, P.32-38 \& Gogate P, 2007, P.965-968). Therefore, the small incision operation is very popular in the Indian blindness prevention operations.

India pays more attention to the training of surgery doctors, especially the training of the local doctors. For the local doctors, the small incision operation is one of main courses, and because of its advantages such as low cost, low risk, high quality, and easy operation, it is very welcomed in the blindness prevention operations, and the free patients are all use this technology.

\section{Cube blindness prevention mode}

The medical treatment is developed in Cuba, and in the planned economic system, the medical treatment of Cuba is advantageous beyond comparison with socialism characteristics. The government would invest large numerous human and materials resources for the blindness prevention, so the blindness prevention of Cube is famous in the world.

\subsection{Medical system}

The blindness prevention advantage of Cuba comes from the medical system of Cuba, i.e. a free medical guarantee system with wide radiation to every household in the whole country.

The free treatment for all people means that the people of Cuba could enjoy the possible free medical service from the country. In the ophthalmologic domain, the patients could freely enjoy the medical treatment level in all ophthalmologic hospitals. Needless to say these ophthalmologic diseases such as cataract, glaucoma, and eye-ground disease, the refractive surgeries to treat the short sight and the hyperopia by the quasi-molecule laser have been popularized to everyone, and only if the patients would accept the surgery, they could completely enjoy the service with high technology. Therefore, in Cuba, it is hard to see the eye diseases which could not be treated for a long time in Cuba, and the surgery of the hypermature cataract is very rare.

Wide radiation range means that the "three-class medical" system of Cube could radiate the medical service to every family. The "three-class medical" system is composed by three classes, i.e. the primary class, the middle class, and the high class, and the primary medical units include the medical stations established in villages and communities. One medical station could cover about 200 600 residents, which is charged by one doctor and one nurse for 24 hours, and each resident has detailed medical file in the medical station, and medical personnel could provide healthy checking, simple disease treatment and disease prevention. If the patient with eye disease is found in the medical station and needs to be further treated, the doctor will help him to the special hospital for treatment. In this way, the asymptomatic eye diseases such as diabetes and glaucoma could be discovered in the physical checking and transferred to the list of early diagnosis and treatment, and the avoidable blindness could be eliminated. And the cataract patients could be treated as soon as possible.

\subsection{Large government investment and numerous oculists}

In recent years, the large-scale blindness prevention work of Cube is very famous in the world. With the basic medical guarantee, Cuba has basically eliminated the avoidable blindness such as cataract, and the blindness prevention work has been ascended to the stage of prevention. Aiming at the avoidable blindness patients, their files would be established in the corresponding medical institutions, and they will be treated and interviewed periodically. At the same time, Cuba also invested many capitals for the blindness prevention in the world. In 2004, Mr. Castro presided in the "Miracle Plan" in person to treat the eye diseases in the Latin America and Caribbean. In this plan was implemented in 2004, and Cuba has established the ophthalmologic hospitals in about 50 countries, and most of them were centralized in the Latin America and Africa, and the third world countries, and from 2005 to 2008 , there were 1.5 million patients to be treated by the surgery.

So much large foreign assistance plan could not leave the quick development of the medical education, and in 2004, there were only less than 700 oculists in Cuba, and in 2010, this number ascended to 2000, and for the country with 12 million population, it means there is one oculist in 6000 people (there is one oculist in 60000 people in China).

\section{The actuality of the blindness prevention in China}

China is the developing country, and there are about 5 million cataract blinds in China (Zhao, 1999, P.329-335), $18 \%$ of the blinds in the world. The yearly cataract surgery rate is only $448.7,1 / 8$ of the number in India, which 
is largely different in similar economic states, saying nothing of comparing with the blindness prevention of Cuba. With the increase of the population and the advent of the aging society, though China is strengthening the power and adopting various measures to strengthen the blindness prevention, the operation amount each year could only achieve the amount of the new patient, which could not solve the problem in essential.

\subsection{Poor people}

The cataract patients in China are mainly centralized in the back lands, and they are too poor to accept the operation, which is the main limited factor of the cataract operation. At present, about $40 \% \sim 60 \%$ people could not pay the charge of the medical treatment in rural China, and in the middle and west regions, $60 \% \sim 80 \%$ patients would die at home because of poor conditions.

\subsection{Sufferers have not paid more attention to the eye diseases}

Chinese people always pay less attention to the eye diseases, and the screening work is rare, so many patients examine only when they find the disease, and many diseases are too late to be treated, and the treatment opportunity has been lost, so is the glaucoma.

\subsection{Deficient and uneven doctors}

According to the statistics, there are 22 thousand oculists in China, one in 60 thousand people, and most of these oculists are centralized in large and middle cities, and the medical power in the rural and back lands is very weak, and about 1/3 counties in China have not oculists (He, 2005, P.121-123). Oculists' whole technology is lower, and less of the half could do the cataract surgery (Zhang, 2006, P.31-32). Facing large numerous of blinds, many oculists in large hospitals go to back lands for various blindness prevention activities such as the "Health Flier", the "Sunshine Travel", and the "Project Vision", with the helps from various castes and charity institutions. But the power is still too limited to solve numerous blinds.

\section{Revelations}

\subsection{Supports from government and society}

As viewed from the economic states and social systems, China has both part of advantages of India and Cuba, and these two countries belong to the third world countries, with undeveloped economy and low average income, but their blindness preventions are better than China. The cross subsidization method of India comes from the love to the society. Rich people would provide part charge for those poor patients to treat the blindness, and the hospitals would abandon the profitable will to help people as they can, and offer the opportunities of screening and treatment. The blindness prevention of Cuba completely comes from the government behaviors, though the finance of the government is not abundant, but it could take much capital for the medical security to perfect the basic medical system, medical education, physical checking, and blindness prevention. Therefore, the blindness prevention of Cuba doesn't only aim at those avoidable eye diseases such as cataract, and the prevention of the preventable blindness could also achieve certain level. Therefore, the work of blindness prevention is not only the work of the ophthalmology, and it represents the basic medical level of one country, and the country quality of one country. It is far sufficient to only depend on ophthalmologic staffs' passions and professional dedications. It needs the care from the government, and the government should increase the investment and the construction of the grassroots medical establishments, perfect the medical system, and make the medical services to every family. With the gradual perfection of the Chinese medical security system, a part of special capitals in the urban and village medical security system and the new rural cooperative medical scheme should be fixed for the blindness prevention. At the same time, the drumbeating of the blindness prevention should be strengthened, and the organization and arrangement of the charities should be increased to introduce more supports and love from all circles of the society.

\subsection{Strengthening the trading of the oculists}

One main cause to limit the cataract surgery is the deficiency of good oculists. Most good oculists with abundant experiences are centralized in large hospitals in big cities, and the grassroots oculists are deficient, but most blindness prevention operations are assumed by the grassroots oculists and young doctors in big hospitals, so the operation quality is the focus problem. In 0.75 million cataract operations from 1988 to 1996, 11\% of the vision after the operation is still lower than 0.3 (Guan, 2006, P.7-9). In addition, in the survey about the vision after cataract operation in Beijing Shunyi, Zhao Jialiang et al found that only 25\% patients' daily life vision exceeded 0.3 (Zhao, 1999, P.329-335). It is obvious that it is very important to strengthen grassroots and young doctors' operation training. The Indian experiences told us that the small incision operation had low cost and risk, and the doctors with a few intraocular operation experiences could completely grasp this technology through short-term training, so the small incision operation was deserved to be extended in the back lands. If the operation training 
bases such as the Aravind Hospital could be established in the whole country depending on big hospitals, the grassroots oculists could be trained systematically, especially for the small incision operations, the quality and quantity of the cataract operation in China could be really enhanced under strictly eligible standards.

\subsection{The blindness preventions in city and college should be different}

The difference of the rural and urban medical conditions is large, and the blindness prevention works should be different. For the cities with better medical conditions, the eye diseases such as cataract are relatively easy to be solved, and the screening and early prevention and treatment of the avoidable blindness diseases such as glaucoma and diabetes retina pathological changes should be strengthened, and in China, Xu Liang et al used the remote image transmission to develop the screening and prevention of the eye diseases in the city (Xu, 2009, P.1-3). For villages, the cataract is the main blindness factor, and many people are blind because of it and lose the living ability. Therefore, the screening and treatment of these avoidable blinds could be strengthened to make them see the sunshine as soon as possible, because it is very important to make all people enjoy the right of the sunshine.

\section{References}

Gogate P, Deshpande M, Nirmalan PK. (2007). Why do phacoemulsification? Manual small-incision cataract surgery is almost as effective, but less expensive. Ophthalmology, 2007 May. No. 5. P. 965-968.

Guan, Huaijin. (2006). How to improve the quantity and visual quality of cataract surgery in China. Ophthalmology in China, No.1. P.7-9.

He, Wei, Xu, Ling \& Zhang, Xin. (2005). Nonphacoemulsification Small Incision Extra Capsular Cataract Extraction for the National Situation of China. Chinese Journal of Practical Ophthalmology. No.2. P.121-123.

Ruit S, Tabin G, Chang D, et al. (2007). A prospective randomized clinical trial of phacoemulsification vs manual sutureless small-incision extracapsular cataract surgery in Nepal. Am J Ophthalmol. 2007 Jan. No.1. P.32-38.

$\mathrm{Xu}$, Liang. (2009). The advantages of ophthalmology and blindness prevention in health reforms. Chinese Hospital Management, No.1. P.1-3.

Xu, Qing \& Wu, Haiyang. (2008). Discussion of Brief Training Model for Cataract Surgery in India. Journal of Practical Preventing Blind, No.2. P.1-3.

Zhang, Wenbin, Xu, Haifeng \& Jia, Dandan. (2006). Investigation of the Status of Ophthalmology Human Resource in Integrated Hospital of China. Chinese Hospital Management, No.1. P.31-32.

Zhao, Jialiang. (1999). Developing the Blindness Prevention and Treatment High-effectively. Chinese Journal of Ophthalmology, No.35. P.329-335. 